OPEN ACCESS

Edited by:

Chih-Ho Lai,

Chang Gung University, Taiwan

Reviewed by:

Maryam Dadar,

Razi Vaccine and Serum Research

Institute, Iran

Kelly Grace Magalhaes,

University of Brasilia, Brazil

Elsa Beatriz Damonte,

University of Buenos Aires, Argentina

*Correspondence:

Dmitri Sviridov

dmitri.sviridov@baker.edu.au

Specialty section

This article was submitted to

Microbial Immunology,

a section of the journal

Frontiers in Immunology

Received: 20 June 2020

Accepted: 27 August 2020

Published: 29 September 2020

Citation:

Sviridov D, Miller YI, Ballout RA,

Remaley AT and Bukrinsky M (2020)

Targeting Lipid Rafts - A Potential

Therapy for COVID-19.

Front. Immunol. 11:574508.

doi: 10.3389/fimmu.2020.574508

\section{Targeting Lipid Rafts-A Potential Therapy for COVID-19}

\author{
Dmitri Sviridov ${ }^{1,2 \star}$, Yury I. Miller ${ }^{3}$, Rami A. Ballout ${ }^{4}$, Alan T. Remaley ${ }^{4}$ and \\ Michael Bukrinsky ${ }^{5}$
}

${ }^{1}$ Baker Heart and Diabetes Institute, Melbourne, VIC, Australia, ${ }^{2}$ Department of Biochemistry and Molecular Biology, Monash University, Clayton, VIC, Australia, ${ }^{3}$ Department of Medicine, University of California, San Diego, La Jolla, CA, United States, ${ }^{4}$ Lipoprotein Metabolism Section, Translational Vascular Medicine Branch, National Heart, Lung and Blood Institute (NHLBI), National Institutes of Health, Bethesda, MD, United States, ${ }^{5}$ Department of Microbiology, Immunology and Tropical Medicine, School of Medicine and Health Sciences, The George Washington University, Washington, DC, United States

COVID-19 is a global pandemic currently in an acute phase of rapid expansion. While public health measures remain the most effective protection strategy at this stage, when the peak passes, it will leave in its wake important health problems. Historically, very few viruses have ever been eradicated. Instead, the virus may persist in communities causing recurrent local outbreaks of the acute infection as well as several chronic diseases that may arise from the presence of a "suppressed" virus or as a consequence of the initial exposure. An ideal solution would be an anti-viral medication that (i) targets multiple stages of the viral lifecycle, (ii) is insensitive to frequent changes of viral phenotype due to mutagenesis, (iii) has broad spectrum, (iv) is safe and (v) also targets co-morbidities of the infection. In this Perspective we discuss a therapeutic approach that owns these attributes, namely "lipid raft therapy." Lipid raft therapy is an approach aimed at reducing the abundance and structural modifications of host lipid rafts or at targeted delivery of therapeutics to the rafts. Lipid rafts are the sites of the initial binding, activation, internalization and cell-to-cell transmission of SARS-CoV-2. They also are key regulators of immune and inflammatory responses, dysregulation of which is characteristic to COVID-19 infection. Lipid raft therapy was successful in targeting many viral infections and inflammatory disorders, and can potentially be highly effective for treatment of COVID-19.

Keywords: COVID-19, SARS-CoV-2, coronavirus, lipid rafts, inflammation, lipids, cholesterol

\section{INTRODUCTION}

COVID-19 is the biggest global pandemic of the 21st century and it may not be the last. Increased interactions of humans with animals amplifies chances of animal viruses "jumping" to humans, while increased density of human population and abundant international travel further contribute to the extremely fast spread of the infectious diseases around the globe. At the peak of a pandemic, public health measures provide the most effective protection against spread of the infection, but when the peak passes, it leaves behind important problems. First, the virus continues circulating in the population causing clusters of outbreaks and "second waves." Second, initial infection often causes an outbreak of various chronic diseases ensuing from the presence of a "suppressed" virus 
or as a long-term consequence of the initial exposure. Global immunization provides a radical solution, but full eradication of an infectious disease has been achieved only a few times throughout history. Many viruses are resistant to vaccination through rapid mutagenesis, like influenza viruses (1), employing cell-to-cell transmission altogether bypassing exposure to antibodies, like HTLV (2), gaining entry through the respiratory tract delaying the access of antibodies to the site of infection, like coronaviruses (3), or a combination of these and yet unknown factors, such as with HIV and influenza. Development of an effective vaccine against coronavirus is challenging; while animal vaccines exist, no human vaccine against any of coronaviruses has been developed so far (4). Furthermore, due to animal origin of SARS$\mathrm{CoV}-2$, existing and new animal reservoirs will provide plentiful opportunities for the virus to mutate and reemerge. Collectively, it makes it unlikely that this virus will be eradicated through vaccination, at least in the short and medium terms. A solution is to develop an anti-viral medication. Ideally, such treatment should target an early stage of the viral lifecycle, be insensitive to frequent changes of viral phenotype due to mutations (e.g., targeting host cell rather than the virus) and be safe. Preferably, this treatment should also target complications of the infection. In this Perspective, we discuss an approach that fulfills these requirements, "lipid raft therapy," that potentially can be applied for treatment of COVID-19.

\section{LIPID RAFTS AND VIRAL INFECTIONS}

Lipid rafts are solid domains of plasma membrane embedded into predominantly fluid membrane (5). Proteins that work together (e.g., in multiunit receptors or endocytosis machinery) are usually located in lipid rafts preventing these molecules from drifting apart, instead keeping them in proximity to each other. Lipid rafts host many receptors involved in immune and inflammatory responses and play a key role in regulation of inflammation (6), an important attribute given the role of unique pattern of immune and inflammatory responses in the clinical manifestations of COVID-19 $(7,8)$. At the same time, numerous viruses, e.g., HIV and Influenza virus, use host lipid rafts as a "point of entry," owing to rafts harboring high concentration of receptors utilized to bind and guide pathogen, as well as affiliated endocytosis machinery ready to take an obligate intracellular parasite inside. Lipid rafts also serve as a platform for pathogen's assembly (e.g., HIV) and as a "point of exit" [Ebola virus, HIV and HBV (9)]. Furthermore, viruses often exploit host raft-associated pathways and modify lipid rafts through binding to rafts and/or releasing raft-modifying factors to further promote their infection cycle. The list of viruses where disruption of rafts was shown to inhibit virus infectivity is long and includes HIV, HCV, Influenza A, Ebola, Marburg and many other viruses [for review see $(9,10)]$; SARS-CoV-2 may also be one of such viruses. It is however important to recognize that despite a success of this experimental approach, so far, no drug acting principally through disruption of lipid rafts has been approved for clinical use as an antiviral treatment.

\section{LIPID RAFTS AND PATHOGENESIS OF SARS-CoV-2}

Molecular pathogenesis of SARS-CoV-2 is schematically presented in Figure 1. SARS-CoV-2 is very similar to its close relative SARS-CoV and pathogenic pathways of both viruses interact with pathways of cellular cholesterol metabolism (11). Both viruses carry a spike (S) protein in their envelope, which is essential for entry into the host cells (12). The S protein docks the viral particle onto angiotensin-converting enzyme 2 (ACE2) (12,13), a membrane protein particularly abundant in the plasma membrane of type II pneumocytes, nasal goblet secretory cells and enterocytes $(12,14)$. ACE2 is a lipid raft protein; disruption of lipid rafts prevents its correct exposure making it impossible for the virus to dock $(15,16)$. After binding to ACE2, the S protein must undergo enzymatic conversion (activation) by either the transmembrane serine protease 2, TMPRSS2, or furin (12). The exact location of these proteases on the plasma membrane is unknown, however, TMPRSS2 co-localizes with ACE2 and is potentially palmitoylated (17), indicating likely lipid raft localization. Cleavage-induced conformational change in the $\mathrm{S}$ protein and ACE2 allows the host cell membrane to invaginate, which is essential for initiating endocytic viral entry. Endocytic and fusion pathways used by SARS-CoV to enter the cell rely on a lipid raft-specific machinery and lipid-raft localization is essential for them to function (18). After internalization, the virus undergoes intracellular trafficking within endosomes, which eventually fuse with mature lysosomes. Within the lysosome, the $S$ protein undergoes another series of enzymatic cleavages and modifications, followed by release of the viral RNA genome into the host cytoplasm (18). Furthermore, an important feature of both SARS-CoV (19) and SARS-CoV-2 (20) is an ability for cell-to-cell transmission, which allows the virus to escape contact with antibody. Cell-to-cell transmission through formation of channels or syncytia requires intact lipid rafts (21). Thus, at least four stages of SARS-CoV-2 lifecycle, initial binding, activation, internalization and cell-to-cell transmission, require intact host rafts to proceed (Figure 1). It follows that targeting host lipid rafts may be an effective strategy to reduce infectivity of SARS-CoV-2, and this was experimentally shown in vitro for SARS-CoV (22).

\section{RAFT THERAPEUTICS}

Targeting lipid rafts for treatment of various diseases, from neurodegeneration and neuropathic pain to cancer, infections and atherosclerosis, is a rapidly growing therapeutic approach, as described in detail in our recent review (23). Fundamentally, there are two ways to utilize lipid rafts for anti-viral therapy, either to directly disrupt these domains, or to use lipid raft for targeted delivery of anti-viral therapeutics. As only approaches involving lipid raft disruption have been investigated in the context of SARS-CoV-2 pathogenesis (Figure 1), we will primarily focus on this mechanism.

One approach is to reduce abundance of lipid rafts by depleting them of lipids responsible for their stability, cholesterol 


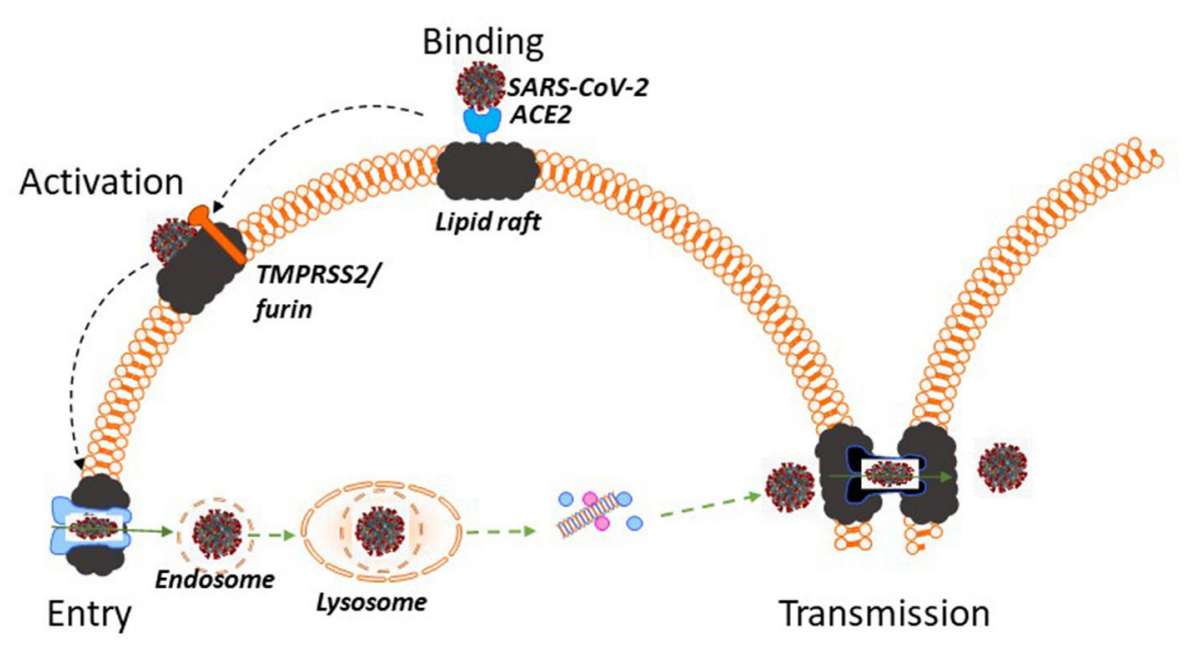

FIGURE 1 | Lipid rafts and pathogenesis of SARS-CoV-2. SARS-CoV-2 docks onto ACE2, which is a lipid raft protein. After binding to ACE2 the S protein in the viral envelope undergoes enzymatic activation by TMPRSS2 or furin, which are likely located in lipid rafts. Subsequent endocytosis of SARS-CoV-2 occurs using raft-dependent endocytic pathway. After internalization SARS-CoV-2 undergoes intracellular trafficking within endosomes, fuses with mature lysosomes and releases its viral RNA genome into the host cytoplasm. One of the pathways of virus transmission, cell-to-cell transmission, occurs through formation of intercellular channels or syncytia and also requires intact lipid rafts. Thus, at least four stages of SARS-CoV-2 lifecycle, initial binding, activation, internalization and cell-to-cell transmission, require intact host rafts to proceed and, if other viruses are a guide, disruption of lipid rafts using lipid raft therapy mitigates the infection.

or sphingomyelin. For example, cholesterol can be physically removed from rafts by sequestrants, such as cyclodextrins. $\beta$ Cyclodextrin is used as a food additive and is proven to be safe for consumption (24), it inhibits entry of another enveloped virus, HIV, into host cells (25) and has antiinflammatory properties (26). Another option is stimulation of physiological pathways responsible for the efflux of cholesterol, such as stimulation of the expression of ATP-binding cassette transporters A1 and G1, using LXR agonists or heterologous over-expression of these transporters. Further, elevation of the levels of natural acceptors of cholesterol, high-density lipoprotein (HDL) and apolipoprotein A-I (apoA-I), or infusion of HDL and apoA-I mimetics also reduces the abundance of lipid rafts. The third option is to reduce cholesterol and sphingolipid supply using inhibitors of their biosynthesis, e.g., statins and Miglustat, respectively. Given the proven safety record of statins, drugs widely used to treat hypercholesterolaemia, they are a good candidate for immediate testing for this application. Finally, recently discovered modulator of lipid rafts, apoA-I Binding Protein (AIBP), may be an especially beneficial "lipid raft therapy" compound to treat COVID-19. AIBP stimulates cholesterol efflux and reduces the abundance of lipid rafts in various tissues (27-29), it effectively reduces inflammation (29) and was recently shown to have an anti-HIV activity (30). Importantly, AIBP targets only cells activated with LPS, cholesterol-loaded or infected, reducing the abundance of lipid rafts to the "healthy level," but not below $(28,30)$, a selectivity beneficial for avoiding adverse side-effects. Another advantage of AIBP is that it remains active when administered directly to lungs via inhalation (31), an application especially relevant to COVID-19.

Second approach takes advantage of unique partitioning of various receptor assemblies to the lipid rafts allowing for an effective targeting of therapeutic agents specifically to these domains. Lipid-coated nanoparticles (32) or liposomes carrying a vector (e.g., antibody to ACE2 or another raft protein) can carry anti-viral therapies, such as Irbesartan, an ACE2 antagonist, Camostat mesylate, a TMPRSS2 inhibitor, or heparin, which breaks down proteoglycans essential for the SARS-CoV-2 binding to the cell, thus increasing the treatment efficiency and reducing the effective drug concentration and toxicity. Finally, $\beta$ cyclodextrin $(33)$ and synthetic $\operatorname{HDL}(34,35)$ particles can also be used to deliver to cells compounds with limited solubility, such as Remdesivir.

\section{LIPID RAFTS AND CO-MORBIDITIES OF COVID-19}

One of the puzzling features of COVID-19 infection is abnormal immune response (7) and severe inflammation resembling autoimmune vasculitis $(36,37)$ or sepsis (38). The exact pathogenesis of dysregulation of the immune and inflammatory responses in COVID-19 is unknown and emergency response relies on general anti-inflammatory medications or inhibitors of inflammatory cytokines. These approaches in most cases are effective in mitigating the acute phase, but, if sepsis and autoimmune vasculitis are a guide, heightened chronic inflammation will persist for a long time causing multiple chronic co-morbidities. Lipid rafts play a key role in immunity and inflammation (6) and targeting lipid rafts to reduce inflammation is a promising therapeutic approach that can reduce both acute and chronic inflammatory responses (23). Another common co-morbidity of COVID-19 is coagulopathy (39). Lipid rafts are involved in regulation of platelet function and lipid raft therapy was effective in attenuating platelet function (40). Thus, therapeutic approaches targeting lipid rafts may 
mitigate both COVID-19 infection itself and its acute and chronic co-morbidities.

\section{DISCUSSION}

Use of "lipid raft therapy" approach has important advantages over other strategies. First, it targets the element of pathogenesis common to both acute and residual infection, as well as the most likely co-morbidities of COVID-19. Second, lipid rafts are a cellular element resistant to rapid mutagenesis, their involvement in pathogenesis of viral infection is common to many viruses and the proposed treatments may be applicable for both current and future pandemics. Third, it utilizes medications that are currently used or being tested for treatment of other diseases, where therapeutic doses were proven to be safe and have high degree of hemocompatibility promising a rapid repurposing for treatment of COVID-19. Finally, it can be used both as a stand-alone therapy and in combination with other therapeutic approaches.

Lipid raft therapy also has some limitations. Agents of lipid raft therapy may trigger immune response, which may dangerously combine with the abnormal immune response to the SARS-CoV-2. Although no adverse immune reaction to any of the lipid raft therapy agents has been described so far (even to large multiprotein complexes such as HDL), such reaction in the context of COVID-19 infection cannot be a priori dismissed. Conversely, disruption of rafts reduces immune responses, which may have a negative effect on resistance to the virus. However, several lines of evidence suggest that suppressed immunity is not detrimental, but could be even beneficial for the antiCOVID-19 therapy. Immunosuppression and chemotherapy did not have a negative effect on severity of COVID-19 (41, 42). Asymptomatic patients have lower levels of virus-specific antibody than those with severe disease manifestation (43). In humans, ACE2, the receptor for SARS-CoV-2, is stimulated by interferon, a key element of anti-viral immune response (14). Collectively, these findings suggest that SARS-CoV-2 is hijacking immune responses, and mitigation of immune response, perhaps

\section{REFERENCES}

1. Altman MO, Angeletti D, Yewdell JW. Antibody immunodominance: the key to understanding influenza virus antigenic drift. Viral Immunol. (2018) 31:142-9. doi: 10.1089/vim.2017.0129

2. Omsland M, Pise-Masison C, Fujikawa D, Galli V, Fenizia C, Parks RW, et al. Inhibition of tunneling nanotube (TNT) formation and human T-cell leukemia virus type 1 (HTLV-1) transmission by cytarabine. Sci Rep. (2018) 8:11118. doi: 10.1038/s41598-018-29391-w

3. He Y, Wang J, Li F, Shi Y. Main clinical features of COVID-19 and potential prognostic and therapeutic value of the microbiota in SARS-CoV-2 infections. Front Microbiol. (2020) 11:1302. doi: 10.3389/fmicb.2020.01302

4. Diamond MS, Pierson TC. The challenges of vaccine development against a new virus during a pandemic. Cell Host Microbe. (2020) 27:699-703. doi: 10.1016/j.chom.2020.04.021

5. Lingwood D, Simons K. Lipid rafts as a membrane-organizing principle. Science. (2010) 327:46-50. doi: 10.1126/science.1174621

6. Sorci-Thomas MG, Thomas MJ. Microdomains, inflammation, and atherosclerosis. Circ Res. (2016) 118:679-91. doi: 10.1161/CIRCRESAHA.115.306246 counterintuitively, may be beneficial. Another limitation is that, given that lipid rafts are a central element of numerous signaling pathways, their disruption may have unintended negative consequences for functionality of these pathways. However, this complication is more of a theoretical nature: while phenotype of excessive raft abundance has well-described manifestations, very little is known of a phenotype of lipid raft deficiency (23), most likely due to a high level of redundancy in regulation of lipid raft originating pathways. Furthermore, some therapeutic approaches, such as AIBP, allows "fine tuning" of the rafts, reducing the overabundant lipid rafts to the "normal" level, but not below $(28,30)$. Finally, some agents of lipid raft therapy may have side-effects seemingly unrelated to their activity toward lipid rafts. For example, high doses of hydroxypropyl- $\beta$-cyclodextrin had unexpected impact on renal and systemic hemodynamics (44). These potential toxicities should be carefully considered when designing therapeutic regimens.

\section{AUTHOR'S NOTE}

After this paper has been accepted for publication, the following article has been published also emphasizing the role of lipid rafts in the pathogenesis of COVID-19 (45).

\section{AUTHOR CONTRIBUTIONS}

DS conceptualized the manuscript and wrote the first draft. $\mathrm{YM}, \mathrm{RB}, \mathrm{AR}$, and $\mathrm{MB}$ contributed ideas and edited subsequent versions on the text. All authors contributed to the article and approved the submitted version.

\section{FUNDING}

This study was supported by grants from the National Institutes of Health: HL131473 (DS and MB), P30 AI117970 (MB), NS102432, HL135737, HL136275, NS104769 (YM) and NIH intramural research funds (RB and $\mathrm{AR}$ ).

7. Giamarellos-Bourboulis EJ, Netea MG, Rovina N, Akinosoglou K, Antoniadou A, Antonakos N, et al. Complex immune dysregulation in COVID-19 patients with severe respiratory failure. Cell Host Microbe. (2020) 27:992-1000.e1003. doi: 10.1016/j.chom.2020.04.009

8. Ong EZ, Chan YFZ, Leong WY, Lee NMY, Kalimuddin S, Haja Mohideen SM, et al. A dynamic immune response shapes COVID-19 progression. Cell Host Microbe. (2020) 27:879-82.e872. doi: 10.1016/j.chom.2020. 03.021

9. Bukrinsky MI, Mukhamedova N, Sviridov D. Lipid rafts and pathogens: the art of deception and exploitation. J Lipid Res. (2020) 61:601-10. doi: 10.1194/jlr.TR119000391

10. Bagam P, Singh DP, Inda ME, Batra S. Unraveling the role of membrane microdomains during microbial infections. Cell Biol Toxicol. (2017) 33:42955. doi: 10.1007/s10565-017-9386-9

11. Ballout RA, Sviridov D, Bukrinsky MI, Remaley AT. The lysosome: a potential juncture between SARS-CoV-2 infectivity and Niemann-Pick disease type C, with therapeutic implications. FASEB J. (2020) 34:7253-64. doi: 10.1096/fj.202000654R

12. Hoffmann M, Kleine-Weber H, Schroeder S, Krüger N, Herrler T, Erichsen $S$, et al. SARS-CoV-2 cell entry depends on ACE2 and TMPRSS2 and is 
blocked by a clinically proven protease inhibitor. Cell. (2020) 181:27180.e278. doi: 10.1016/j.cell.2020.02.052

13. Hofmann H, Pohlmann S. Cellular entry of the SARS coronavirus. Trends Microbiol. (2004) 12:466-72. doi: 10.1016/j.tim.2004.08.008

14. Ziegler CGK, Allon SJ, Nyquist SK, Mbano IM, Miao VN, Tzouanas CN, et al. SARS-CoV-2 receptor ACE2 is an interferon-stimulated gene in human airway epithelial cells and is detected in specific cell subsets across tissues. Cell. (2020) 181:1016-35 e1019. doi: 10.1016/j.cell.2020.04.035

15. Glende J, Schwegmann-Wessels C, Al-Falah M, Pfefferle S, Qu X, Deng $\mathrm{H}$, et al. Importance of cholesterol-rich membrane microdomains in the interaction of the $\mathrm{S}$ protein of SARS-coronavirus with the cellular receptor angiotensin-converting enzyme 2. Virology. (2008) 381:215-21. doi: $10.1016 /$ j.virol.2008.08.026

16. Lu Y, Liu DX, Tam JP. Lipid rafts are involved in SARS-CoV entry into Vero E6 cells. Biochem Biophys Res Commun. (2008) 369:344-9. doi: 10.1016/j.bbrc.2008.02.023

17. Shulla A, Heald-Sargent T, Subramanya G, Zhao J, Perlman S, Gallagher T. A transmembrane serine protease is linked to the severe acute respiratory syndrome coronavirus receptor and activates virus entry. J Virol. (2011) 85:873-82. doi: 10.1128/JVI.02062-10

18. Wang H, Yang P, Liu K, Guo F, Zhang Y, Zhang G, et al. SARS coronavirus entry into host cells through a novel clathrin- and caveolae-independent endocytic pathway. Cell Res. (2008) 18:290-301. doi: 10.1038/cr.2008.15

19. Li W, Moore MJ, Vasilieva N, Sui J, Wong SK, Berne MA, et al. Angiotensinconverting enzyme 2 is a functional receptor for the SARS coronavirus. Nature. (2003) 426:450-4. doi: 10.1038/nature02145

20. Musarrat F, Chouljenko V, Dahal A, Nabi R, Chouljenko T, Jois SD, et al. The anti-HIV drug nelfinavir mesylate (Viracept) is a potent inhibitor of cell fusion caused by the SARSCoV-2 spike (S) glycoprotein warranting further evaluation as an antiviral against COVID-19 infections. J Med Virol. (2020). doi: 10.1002/jmv.25985. [Epub ahead of print].

21. Niyogi K, Hildreth JE. Characterization of new syncytium-inhibiting monoclonal antibodies implicates lipid rafts in human T-cell leukemia virus type 1 syncytium formation. J Virol. (2001) 75:7351-61. doi: 10.1128/JVI.75.16.7351-7361.2001

22. Li GM, Li YG, Yamate M, Li SM, Ikuta K. Lipid rafts play an important role in the early stage of severe acute respiratory syndrome-coronavirus life cycle. Microbes Infect. (2007) 9:96-102. doi: 10.1016/j.micinf.2006.10.015

23. Sviridov D, Mukhamedova N, Miller YI. Lipid rafts as a therapeutic target. $J$ Lipid Res. (2020) 61:687-95. doi: 10.1194/jlr.TR120000658

24. Mortensen A, Aguilar F, Crebelli R, Di Domenico A, Dusemund B, Frutos MJ, et al. Re-evaluation of $\beta$-cyclodextrin (E 459) as a food additive. EFSA J. (2016) 14:e04628. doi: 10.2903/j.efsa.2016.4628

25. Carter GC, Bernstone L, Sangani D, Bee JW, Harder T, James W. HIV entry in macrophages is dependent on intact lipid rafts. Virology. (2009) 386:192-202. doi: 10.1016/j.virol.2008.12.031

26. Zimmer S, Grebe A, Bakke SS, Bode N, Halvorsen B, Ulas T, et al. Cyclodextrin promotes atherosclerosis regression via macrophage reprogramming. Sci Transl Med. (2016) 8:333ra350. doi: 10.1126/scitranslmed. aad6100

27. Schneider DA, Choi S-H, Agatisa-Boyle C, Zhu L, Kim J, Pattison J, et al. AIBP protects against metabolic abnormalities and atherosclerosis. J Lipid Res. (2018) 59:854-63. doi: 10.1194/jlr.M083618

28. Woller SA, Choi S-H, An EJ, Low H, Schneider DA, Ramachandran R, et al. Inhibition of neuroinflammation by AIBP: spinal effects upon facilitated pain states. Cell Rep. (2018) 23:2667-77. doi: 10.1016/j.celrep.2018.04.110

29. Fang L, Miller YI. Regulation of lipid rafts, angiogenesis and inflammation by AIBP. Curr Opin Lipidol. (2019) 30:218-23. doi: 10.1097/MOL.0000000000000596

30. Dubrovsky L, Ward A, Choi S-H, Pushkarsky T, Brichacek B, Vanpouille C, et al. Inhibition of HIV replication by apolipoprotein AI binding protein targeting the lipid rafts. mBio. (2020) 11:e02956-19. doi: $10.1128 / \mathrm{mBio} .02956-19$

31. Choi S-H, Wallace AM, Schneider DA, Burg E, Kim J, Alekseeva E, et al. AIBP augments cholesterol efflux from alveolar macrophages to surfactant and reduces acute lung inflammation. JCI Insight. (2018) 3:e120519. doi: $10.1172 /$ jci.insight. 120519
32. Partlow KC, Lanza GM, Wickline SA. Exploiting lipid raft transport with membrane targeted nanoparticles: a strategy for cytosolic drug delivery. Biomaterials. (2008) 29:3367-75. doi: 10.1016/j.biomaterials.2008.04.030

33. Lee SY, Ko SH, Shim JS, Kim DD, Cho HJ. Tumor targeting and lipid rafts disrupting hyaluronic acid-cyclodextrin-based nanoassembled structure for cancer therapy. ACS Appl Mater Interfaces. (2018) 10:36628-40. doi: 10.1021 /acsami.8b08243

34. Sabnis S, Sabnis NA, Raut S, Lacko AG. Superparamagnetic reconstituted high-density lipoprotein nanocarriers for magnetically guided drug delivery. Int J Nanomed. (2017) 12:1453-64. doi: 10.2147/IJN.S122036

35. Panja P, Jana NR. Lipid-raft-mediated direct cytosolic delivery of polymer-coated soft nanoparticles. J Phys Chem B. (2020) 124:5323-33. doi: $10.1021 /$ acs.jpcb.0c03444

36. Verdoni L, Mazza A, Gervasoni A, Martelli L, Ruggeri M, Ciuffreda M, et al. An outbreak of severe Kawasaki-like disease at the Italian epicentre of the SARS-CoV-2 epidemic: an observational cohort study. Lancet. (2020) 395:1771-8. doi: 10.1016/S0140-6736(20)31103-X

37. Zhou Z, Ren L, Zhang L, Zhong J, Xiao Y, Jia Z, et al. Heightened innate immune responses in the respiratory tract of COVID-19 patients. Cell Host Microbe. (2020) 27:883-90.e2. doi: 10.1016/j.chom.2020.04.017

38. Li H, Liu L, Zhang D, Xu J, Dai H, Tang N, et al. SARS-CoV-2 and viral sepsis: observations and hypotheses. Lancet. (2020) 395:1517-20. doi: 10.1016/S0140-6736(20)30920-X

39. Miesbach W, Makris M. COVID-19: coagulopathy, risk of thrombosis, and the rationale for anticoagulation. Clin Appl Thromb Hemost. (2020) 26:1076029620938149. doi: 10.1177/10760296209 38149

40. Calkin AC, Drew BG, Ono A, Duffy SJ, Gordon MV, Schoenwaelder $\mathrm{SM}$, et al. Reconstituted high-density lipoprotein attenuates platelet function in individuals with type 2 diabetes mellitus by promoting cholesterol efflux. Circulation. (2009) 120:2095-104. doi: 10.1161/CIRCULATIONAHA.109.870709

41. Koker O, Demirkan FG, Kayaalp G, Cakmak F, Tanatar A, Karadag SG, et al. Does immunosuppressive treatment entail an additional risk for children with rheumatic diseases? A survey-based study in the era of COVID-19. Rheumatol Int. (2020) 40:1613-23. doi: 10.1007/s00296-020-04663-9

42. Robilotti EV, Babady NE, Mead PA, Rolling T, Perez-Johnston R, Bernardes $\mathrm{M}$, et al. Determinants of COVID-19 disease severity in patients with cancer. Nat Med. (2020) 26:1218-23. doi: 10.1038/s41591-020-0979-0

43. Long Q-X, Tang X-J, Shi Q-L, Li Q, Deng H-J, Yuan J, et al. Clinical and immunological assessment of asymptomatic SARS-CoV-2 infections. Nat Med. (2020) 26:1200-4. doi: 10.1038/s41591-020-0965-6

44. Rosseels ML, Delaunois AG, Hanon E, Guillaume PJ, Martin FD, Van Den Dobbelsteen DJ. Hydroxypropyl- $\beta$-cyclodextrin impacts renal and systemic hemodynamics in the anesthetized dog. Regul Toxicol Pharmacol. (2013) 67:351-9. doi: 10.1016/j.yrtph.2013.08.013

45. Fecchi K, Anticoli S, Peruzzu D, Iessi E, Gagliardi MC, Matarrese P, Ruggieri A. Coronavirus Interplay with lipid rafts and autophagy unveils promising therapeutic targets. Front. Microbiol. (2020) 11:1821.

Conflict of Interest: DS, YM, and MB are inventors listed in patent applications related to the topic of this paper. YM is scientific co-founder of Raft Pharmaceuticals LLC. The terms of this arrangement have been reviewed and approved by the University of California, San Diego in accordance with its conflict of interest policies.

The remaining authors declare that the research was conducted in the absence of any commercial or financial relationships that could be construed as a potential conflict of interest.

Copyright $\odot 2020$ Sviridov, Miller, Ballout, Remaley and Bukrinsky. This is an openaccess article distributed under the terms of the Creative Commons Attribution License (CC BY). The use, distribution or reproduction in other forums is permitted, provided the original author(s) and the copyright owner(s) are credited and that the original publication in this journal is cited, in accordance with accepted academic practice. No use, distribution or reproduction is permitted which does not comply with these terms. 